\title{
TENSOR PRODUCT CONSTRUCTION OF 2-FREENESS
}

\author{
R. LENCZEWSKI \\ Institute of Mathematics, Technical University of Wroctaw \\ Wybrzeże Wyspiańskiego 27, 50-370 Wroctaw, Poland \\ E-mail: lenczew@im.pwr.wroc.edu.pl
}

\begin{abstract}
From a sequence of $m$-fold tensor product constructions that give a hierarchy of freeness indexed by natural numbers $m$ we examine in detail the first non-trivial case corresponding to $m=2$ which we call 2 -freeness. We show that in this case the constructed tensor product of states agrees with the conditionally free product for correlations of order $\leq 4$. We also show how to associate with 2 -freeness a cocommutative ${ }^{*}$-bialgebra.
\end{abstract}

1. Introduction. Recently we have made an attempt to treat the conditionally free product of free *-algebras by means of appropriately extended *-algebras and their $m$ fold tensor products. Our approach gives the Boolean case for $m=1$ which in the new terminology corresponds to 1 -freeness. This case is fairly straightforward, the first really non-trivial one being that of 2 -freeness. For that purpose we need a 2 -fold tensor product construction. We originally thought that the 2 -fold tensor product would give the conditionally free product. However, later we realized that it worked for correlations of order $\leq 4$ and successive iterations are needed to recover the conditionally free case. This leads to a sequence of $m$-fold tensor product constructions that give a hierarchy of freeness which in the limit gives (conditional) freeness. Nevertheless, the 2-freeness is a model case and provides a nice introduction to the general theory of the hierarchy of freeness and proofs of many facts simplify considerably. The general case will be treated elsewhere. We should add that we often use the term freeness associated with the Voiculescu theory [V-D-N] instead of conditional freeness [B-L-S] for simplicity as well as due to the fact that from the point of view of our approach there is no essential difference between them.

In our approach, instead of making the product noncommutative in the definition of the product of free ${ }^{*}$-algebras, we stick to the tensor product and take noncommutative extensions of those *-algebras with non-canonical embeddings. The idea consists in finding appropriate deformations of the canonical embeddings. In the Boolean case for instance, if $\mathcal{A}_{1}, \mathcal{A}_{2}$ are free ${ }^{*}$-algebras, then we define their extensions $\tilde{\mathcal{A}}_{1}, \tilde{\mathcal{A}}_{2}$, respectively, where

1991 Mathematics Subject Classification: Primary 46L50; Secondary 81R50.

The paper is in final form and no version of it will be published elsewhere. 
$\tilde{\mathcal{A}}_{k}=\mathcal{A}_{k} * \mathbf{C}[t]$ and $t^{*}=t$. Then, instead of the canonical embeddings

$$
i_{k}: \mathcal{A}_{k} \rightarrow \mathcal{A}_{1} \otimes \mathcal{A}_{2}, \quad k=1,2,
$$

given by

$$
i_{1}(w)=w \otimes 1, \quad i_{2}(v)=1 \otimes v
$$

where $w, v$ are words in $\mathcal{A}_{1}, \mathcal{A}_{2}$, respectively, we take

$$
\begin{gathered}
j_{k}^{(1)}: \mathcal{A}_{k} \rightarrow \tilde{\mathcal{A}}_{1} \otimes \tilde{\mathcal{A}}_{2}, \\
j_{1}^{(1)}(w)=w \otimes t^{l(w)}, \quad j_{2}^{(1)}(v)=t^{l(v)} \otimes v,
\end{gathered}
$$

where $l(w), l(v)$ denote the lengths of words $w, v$, and extend it to $\mathcal{A}_{1} * \mathcal{A}_{2}$ homomorphically:

i.e. $j^{(1)}=1 \otimes 1$ and

$$
j^{(1)}: \mathcal{A}_{1} * \mathcal{A}_{2} \rightarrow \tilde{\mathcal{A}}_{1} \otimes \tilde{\mathcal{A}}_{2},
$$

$$
j^{(1)}\left(w_{1} \ldots w_{n}\right)=j_{k_{1}}^{(1)}\left(w_{1}\right) \ldots j_{k_{n}}^{(1)}\left(w_{n}\right)
$$

where $w_{1}, \ldots, w_{n} \in \mathcal{A}_{k_{1}}, \ldots, \mathcal{A}_{k_{n}}$ and $k_{1} \neq k_{2} \neq \ldots \neq k_{n}, k_{1}, \ldots, k_{n} \in\{1,2\}$.

Now, if $\phi_{1}, \phi_{2}$ are states on $\mathcal{A}_{1}, \mathcal{A}_{2}$, respectively, then we define their Boolean extensions $\tilde{\phi}_{1}, \tilde{\phi}_{2}$ on $\tilde{\mathcal{A}}_{1}, \tilde{\mathcal{A}}_{2}$, for which

$$
\tilde{\phi}_{k}\left(t_{0} w_{1} t_{1} \ldots w_{n} t_{n}\right)=\phi_{k}\left(w_{1}\right) \ldots \phi_{k}\left(w_{n}\right)
$$

where $w_{1}, \ldots, w_{n}$ are non-empty words in $\mathcal{A}_{k}$ and $t_{0}, \ldots, t_{n}$ are powers of $t$, of which $t_{1}, \ldots, t_{n-1} \neq 1$. If $\tilde{\Phi}^{(1)}=\tilde{\phi}_{1} \otimes \tilde{\phi}_{2}$, then it is easy to see that

$$
\tilde{\Phi}^{(1)}\left(j_{k_{1}}^{(1)}\left(w_{1}\right) \ldots j_{k_{n}}^{(1)}\left(w_{n}\right)\right)=\phi_{k_{1}}\left(w_{1}\right) \ldots \phi_{k_{n}}\left(w_{n}\right),
$$

where $w_{1}, \ldots, w_{n} \in \mathcal{A}_{k_{1}}, \ldots, \mathcal{A}_{k_{n}}$ and $k_{1} \neq k_{2} \neq \ldots \neq k_{n}$. Hence, $\tilde{\Phi}^{(1)} \circ j^{(1)}$ agrees with the Boolean product state.

One can see that the idea is related to the deformations of the canonical embeddings used in the case of $q-^{*}$-bialgebras studied by Schürmann [Sch] and $q$-deformed enveloping algebras investigated in the context of central limit theorems in [Len1-Len2]. However, the free case as well as the general version of the conditionally free case are not so easy to handle. Firstly, one needs to take tensor products of higher order, thus arriving at a sequence of $m$-fold tensor product constructions. Secondly, the embeddings are more complicated. They consist of a number of compensating pairs and the number of terms is growing (it equals $2 m+1$ ). Thirdly, the conditionally free case is obtained in the limit when $m \rightarrow \infty$. In this paper we treat the case $m=2$.

In Section 2, for given two free ${ }^{*}$-algebras $\mathcal{A}_{1}$ and $\mathcal{A}_{2}$, we consider the double tensor product

$$
\tilde{\mathcal{A}}^{(2)}=\tilde{\mathcal{A}}_{1} \otimes \tilde{\mathcal{A}}_{1} \otimes \tilde{\mathcal{A}}_{2} \otimes \tilde{\mathcal{A}}_{2}
$$

where $\tilde{\mathcal{A}}_{i}=\mathcal{A}_{i} * \mathbf{C}[t], i=1,2$, with hermitian $t$. Given two pairs of states on those *-algebras, namely $\left(\phi_{1}, \psi_{1}\right)$ and $\left(\phi_{2}, \psi_{2}\right)$, respectively, we construct the state

$$
\tilde{\Phi}^{(2)} \equiv \tilde{\Phi}_{1}^{(2)} \otimes \tilde{\Phi}_{2}^{(2)}=\tilde{\phi}_{1} \otimes \tilde{\psi}_{1} \otimes \tilde{\phi}_{2} \otimes \tilde{\psi}_{2}
$$

where $\tilde{\Phi}_{i}=\tilde{\phi}_{i} \otimes \tilde{\psi}_{i}, i=1,2$. Then we define a ${ }^{*}$-homomorphism $j^{(2)}$ from $\mathcal{A}_{1} * \mathcal{A}_{2}$ into $\tilde{\mathcal{A}}^{(2)}$ which is one step closer to freeness as compared with $j^{(1)}$. 
In Section 3 we show that $\tilde{\Phi}^{(2)} \circ j^{(2)}$ agrees with the conditionally free product of $\left(\phi_{1}, \psi_{1}\right)$ and $\left(\phi_{2}, \psi_{2}\right)$, denoted by $*_{i \in\{1,2\}}\left(\phi_{i}, \psi_{i}\right)$, on products $w_{1} \ldots w_{n}$ for $n \leq 4$. Thus, $\tilde{\Phi}^{(2)} \circ j^{(2)}$ can be identified with a state of 2 -freeness.

In Section 4 this construction is extended to an infinite number of *-algebras by means of the infinite tensor product of ${ }^{*}$-algebras $\bigotimes_{i \in \mathbf{N}} \tilde{\mathcal{A}}_{i}^{\otimes 2}$.

In Section 5 we restrict ourselves to the case of one free ${ }^{*}$-algebra: $\mathcal{A}_{i}=\mathcal{A}$ for all $i \in \mathbf{N}$. This corresponds to the case of conditionally free convolution of states on $\mathcal{A}$. We equip the free product

$$
\tilde{\mathcal{A}}^{*(2)}=\tilde{\mathcal{A}} * \tilde{\mathcal{A}}
$$

with a *-bialgebra structure $\left(\tilde{\mathcal{A}}^{*(2)}, \Delta^{(2)}, \epsilon^{(2)}\right)$ with coproduct $\Delta^{(2)}$ and counit $\epsilon^{(2)}$.

In Section 6 we make some comments on the generalization of our approach which leads to a hierarchy of freeness and a unification of independence.

2. Preliminaries. By a quantum probability space we shall understand a pair $(\mathcal{A}, \phi)$, where $\mathcal{A}$ is a unital ${ }^{*}$-algebra and $\phi: \mathcal{A} \rightarrow \mathbf{C}$ is a state, i.e. a normalized $(\phi(1)=1)$, positive $\left(\phi\left(x x^{*}\right) \geq 0\right.$ for all $\left.x \in \mathcal{A}\right)$ functional.

Our construction will be carried out for free ${ }^{*}$-algebras $\mathcal{A}$ generated by a set $\mathcal{G}^{+}$. We denote $\mathcal{G}^{-}=\left\{a^{*} \mid a \in \mathcal{G}^{+}\right\}, \mathcal{G}=\mathcal{G}^{+} \cup \mathcal{G}^{-}$. Nonempty words in $\mathcal{A}$ will be denoted by $w=a_{1} \ldots a_{k}$, where $a_{i} \in \mathcal{G}$. The length of $w$ will be denoted by $l(w)$. We allow the empty word, which is denoted by 1 , of length $l(1)=0$. The involution is given by the antilinear extension of $\left(a_{1} \ldots a_{k}\right)^{*}=a_{k}^{*} \ldots a_{1}^{*}$.

For a given free ${ }^{*}$-algebra $\mathcal{A}$ we consider the free product of $\mathcal{A}$ and $\mathbf{C}[t]$, the algebra of polynomials in one hermitian variable $t$, which we denote

$$
\tilde{\mathcal{A}}=\mathcal{A} * \mathbf{C}[t]
$$

In this free product we identify units. Also, we equip $\tilde{\mathcal{A}}$ with a natural involution defined by the antilinear extension of

$$
\left(t_{0} w_{1} t_{1} \ldots w_{n} t_{n}\right)^{*}=t_{n} w_{n}^{*} \ldots t_{1} w_{1}^{*} t_{0},
$$

where $w_{1}, \ldots, w_{n}$ are non-empty words in $\mathcal{A}$, and $t_{0}, \ldots, t_{n}$ are monomials in $\mathbf{C}[t]$, of which $t_{1}, \ldots, t_{n-1} \neq 1$.

Definition 1. For a given state $\phi$ on $\mathcal{A}$, and a state $h$ on $\mathbf{C}[t]$ we define the Boolean extension of $\tilde{\phi}$ on $\tilde{\mathcal{A}}$, denoted $\tilde{\phi}=\phi *_{B} h$, as the linear extension of $\tilde{\phi}(1)=1$ and

$$
\tilde{\phi}\left(t_{0} w_{1} t_{1} \ldots w_{n} t_{n}\right)=h\left(t_{0}\right) \ldots h\left(t_{n}\right) \phi\left(w_{1}\right) \ldots \phi\left(w_{n}\right)
$$

where $w_{1}, \ldots, w_{n}$ are non-empty words in $\mathcal{A}$ and $t_{0}, \ldots, t_{n}$ are monomials in $\mathbf{C}[t]$, of which $t_{1}, \ldots, t_{n-1} \neq 1$.

From $[\mathrm{B}-\mathrm{L}-\mathrm{S}]$ it follows that $\tilde{\phi}$ is a state on $\tilde{\mathcal{A}}$. In this paper we will assume that $h$ is a *-homomorphism and $h(t)=1$.

One can say that the generator $t$ serves as a "Boolean identity", in contrast to $U_{q}(s u(2))$-type Hopf algebras, where a similar object satisfies certain $q$-commutation relation and can be viewed as a "q-identity". In any case, it is convenient to view $t$ as a "twisted identity". Only here, we view it simply as "more noncommutative". Note that 
it plays the role of a separator of words from the ${ }^{*}$-algebra $\mathcal{A}$. This nice property will be crucial in further considerations.

Let us finally recall the definition of the conditionally free product of ${ }^{*}$-algebras. For a given family of unital ${ }^{*}$-algebras $\mathcal{A}_{i}, i \in I$, and given pairs of states $\phi_{i}, \psi_{i}$ on $\mathcal{A}_{i}$, one can define a state $\phi=*_{i \in I}\left(\phi_{i}, \psi_{i}\right)$ on their free product $*_{i \in I} \mathcal{A}_{i}$ by $\phi(1)=1$ and the factorization property

$$
\phi\left(a_{1} \ldots a_{n}\right)=\phi_{k_{1}}\left(a_{1}\right) \ldots \phi_{k_{n}}\left(a_{n}\right),
$$

whenever $a_{j} \in \mathcal{A}_{k_{j}}$ and $\psi_{k_{j}}\left(a_{j}\right)=0$, where $k_{1} \neq k_{2} \neq \ldots \neq k_{n}$. In particular, when $\psi_{j}=\phi_{j}$, we obtain the free independence, and when $\psi_{j}=\pi_{1}$, where $\pi_{1}(1)=1$ and $\pi_{1}(w)=0$ for any non-empty word $w$, we get the Boolean independence.

For given two free ${ }^{*}$-algebras $\mathcal{A}_{1}, \mathcal{A}_{2}$ generated by $\mathcal{G}_{1}^{+}, \mathcal{G}_{2}^{+}$, respectively, let $\mathcal{G}_{i}=\mathcal{G}_{i}^{+} \cup$ $\mathcal{G}_{i}^{-}$, where $\mathcal{G}_{i}^{-}=\left\{a^{*} \mid a \in \mathcal{G}_{i}^{+}\right\}, i=1,2$. Given two pairs of states on those ${ }^{*}$-algebras, namely $\left(\phi_{i}, \psi_{i}\right), i=1,2$, we construct their Boolean extensions $\left(\tilde{\phi}_{i}, \tilde{\psi}_{i}\right)$ on $\tilde{\mathcal{A}}_{i}$, as explained in Section 2. Using those extensions, we will construct a new quantum probability space $\left(\mathcal{A}^{(2)}, \Phi^{(2)}\right)$, where $\mathcal{A}^{(2)}$ is a ${ }^{*}$-subalgebra of the double tensor product

$$
\tilde{\mathcal{A}}^{(2)} \equiv \tilde{\mathcal{A}}_{1} \otimes \tilde{\mathcal{A}}_{1} \otimes \tilde{\mathcal{A}}_{2} \otimes \tilde{\mathcal{A}}_{2}
$$

and $\Phi^{(2)}$ is the restriction of

$$
\tilde{\Phi}^{(2)} \equiv \tilde{\psi}_{1} \otimes \tilde{\phi}_{1} \otimes \tilde{\psi}_{2} \otimes \tilde{\phi}_{2}
$$

to $\mathcal{A}^{(2)}$. The involution on the tensor product is given by $\left(b_{1} \otimes b_{2} \otimes c_{1} \otimes c_{2}\right)^{*}=b_{1}^{*} \otimes b_{2}^{*} \otimes$ $c_{1}^{*} \otimes c_{2}^{*}$. The ${ }^{*}$-subalgebra $\mathcal{A}^{(2)}$ will be the image of the ${ }^{*}$-homomorphism $j^{(2)}$ given in Definition 2. We introduce the following double tensor notation: $T=t \otimes t, T_{2}=1 \otimes t$, $1=1 \otimes 1$.

Definition 2. For given $a \in \mathcal{G}_{1}, b \in \mathcal{G}_{2}$, let

$$
\begin{gathered}
j_{1}^{(2)}(a)=i_{1}(a) \otimes T+i_{2}(a) \otimes\left(T_{2}-T\right) \\
j_{2}^{(2)}(b)=T \otimes i_{1}(b)+\left(T_{2}-T\right) \otimes i_{2}(b)
\end{gathered}
$$

and define the ${ }^{*}$-homomorphism

$$
j^{(2)}: \mathcal{A}_{1} * \mathcal{A}_{2} \rightarrow \tilde{\mathcal{A}}_{1} \otimes \tilde{\mathcal{A}}_{1} \otimes \tilde{\mathcal{A}}_{2} \otimes \tilde{\mathcal{A}}_{2}
$$

as the linear extension of $j^{(2)}(1)=\mathbf{1} \otimes \mathbf{1}$ and

$$
j^{(2)}\left(w_{1} \ldots w_{n}\right)=j_{k_{1}}^{(2)}\left(w_{1}\right) \ldots j_{k_{n}}^{(2)}\left(w_{n}\right)
$$

where $w_{1}, \ldots, w_{n}$ are non-empty words in $\mathcal{A}_{k_{1}}, \ldots, \mathcal{A}_{k_{n}}$, where $k_{1}, \ldots, k_{n} \in\{1,2\}$.

It should be noted that in this definition we do not assume that the neighbouring indices are different.

3. Main results. We need to calculate $j_{k}^{(2)}(w), k=1,2$, where $w$ is a non-empty word in $\mathcal{A}_{i}$. We will use the following notation. Let $w=a_{1} \ldots a_{n}$ and $P=\{1, \ldots, n\}$. If $P^{\prime} \subset P$, let $w_{P^{\prime}}=\prod_{i \in P^{\prime}} a_{i}$, where the product is taken in the natural order. Then, for given $w$, the summation over all pairs of its subwords, $\left(w_{P_{1}}, w_{P_{2}}\right)$ such that $\left\{P_{1}, P_{2}\right\}$ are disjoint (possibly empty) subsets of $P$ and $P_{1} \cup P_{2}=P$, will be written as the summation over $w^{\prime}, w^{\prime \prime}$, where $w^{\prime}=w_{P_{1}}, w^{\prime \prime}=w_{P_{2}}$. 
Proposition 1. Let $w, v$ be non-empty words in $\mathcal{A}_{1}, \mathcal{A}_{2}$, respectively. Then

$$
\begin{gathered}
j_{1}^{(2)}(w)=\sum_{w^{\prime}, w^{\prime \prime}} i_{1}\left(w^{\prime}\right) i_{2}\left(w^{\prime \prime}\right) \otimes T^{l\left(w^{\prime}\right)}\left(T_{2}-T\right)^{l\left(w^{\prime \prime}\right)}, \\
j_{2}^{(2)}(v)=\sum_{v^{\prime}, v^{\prime \prime}} T^{l\left(v^{\prime}\right)}\left(T_{2}-T\right)^{l\left(v^{\prime \prime}\right)} \otimes i_{1}\left(v^{\prime}\right) i_{2}\left(v^{\prime \prime}\right) .
\end{gathered}
$$

Pr o of. We will prove the first identity since the proof of the second one is similar. Thus, let $w=a_{1} \ldots a_{n}$. Then

$$
\begin{aligned}
j_{1}^{(2)}(w) & =\prod_{k=1}^{n}\left(i_{1}\left(a_{k}\right) \otimes T+i_{2}\left(a_{k}\right) \otimes\left(T_{2}-T\right)\right) \\
& =\sum_{k_{1}, \ldots, k_{n} \in\{1,2\}} i_{k_{1}}\left(a_{1}\right) \ldots i_{k_{n}}\left(a_{n}\right) \otimes T^{\left|P_{1}\right|}\left(T_{2}-T\right)^{\left|P_{2}\right|}
\end{aligned}
$$

where $P_{1}=\left\{i \mid k_{i}=1\right\}, P_{2}=\left\{i \mid k_{i}=2\right\}$ and $\left|P_{i}\right|$ denotes the cardinality of $P_{i}, i=1,2$. Since $i_{1}(a) i_{2}\left(a^{\prime}\right)=i_{2}\left(a^{\prime}\right) i_{1}(a)$ for any $a, a^{\prime} \in \mathcal{G}_{1}$, we can write

$$
j_{1}^{(2)}(w)=\sum_{P_{1}, P_{2}} i_{1}\left(w_{P_{1}}\right) i_{2}\left(w_{P_{2}}\right) \otimes T^{\left|P_{1}\right|}\left(T_{2}-T\right)^{\left|P_{2}\right|}
$$

where the summation is taken over all pairs $\left(P_{1}, P_{2}\right)$ of disjoint subsets of $P$ such that $P=P_{1} \cup P_{2}$. Putting $w^{\prime}=w_{P_{1}}, w^{\prime \prime}=w_{P_{2}}$, which implies that $\left|P_{1}\right|=l\left(w^{\prime}\right)$ and $\left|P_{2}\right|=$ $l\left(w^{\prime \prime}\right)$, we obtain the desired result.

It is also useful to give the corresponding formulas in terms of 4 -fold tensor products.

Proposition 2. Under the assumptions of Proposition 1 we have

$$
\begin{gathered}
j_{1}^{(2)}(w)=\sum_{w^{\prime}, w^{\prime \prime}} \sum_{r=0}^{l\left(w^{\prime \prime}\right)}\left(\begin{array}{c}
l\left(w^{\prime \prime}\right) \\
r
\end{array}\right)(-1)^{l\left(w^{\prime \prime}\right)-r} w^{\prime} \otimes w^{\prime \prime} \otimes t^{l(w)-r} \otimes t^{l(w)}, \\
j_{2}^{(2)}(v)=\sum_{v^{\prime}, v^{\prime \prime}} \sum_{r=0}^{l\left(v^{\prime \prime}\right)}\left(\begin{array}{c}
l\left(v^{\prime \prime}\right) \\
r
\end{array}\right)(-1)^{l\left(v^{\prime \prime}\right)-r} t^{l(v)-r} \otimes t^{l(w)} \otimes v^{\prime} \otimes v^{\prime \prime} .
\end{gathered}
$$

Let us now calculate $\tilde{\Phi}^{(2)} \circ j_{k}^{(2)}$ on $\mathcal{A}_{k}, k=1,2$.

Proposition 3. Let $\tilde{\Phi}^{(2)}=\tilde{\psi}_{1} \otimes \tilde{\phi}_{1} \otimes \tilde{\psi}_{2} \otimes \tilde{\phi}_{2}$, where $\tilde{\phi}_{i}(t)=\tilde{\psi}_{i}(t)=h(t)=1$, $i=1,2$. Then

$$
\tilde{\Phi}^{(2)} \circ j_{1}^{(2)}=\phi_{1}, \quad \tilde{\Phi}^{(2)} \circ j_{2}^{(2)}=\phi_{2} .
$$

Proof. Clearly, $\left(\tilde{\Phi}^{(2)} \circ j_{k}^{(2)}\right)(1)=1=\phi_{k}(1)$, since $\phi_{k}, \psi_{k}$ are states on $\mathcal{A}_{k}, k=1,2$. Thus, assume that $w=a_{1} \ldots a_{n}$ is a non-empty word in $\mathcal{A}_{1}$. Using Proposition 1 , we can write

$$
\begin{gathered}
\left(\tilde{\Phi}^{(2)} \circ j_{1}^{(2)}\right)(w)=\tilde{\Phi}^{(2)}\left(\sum_{w^{\prime}, w^{\prime \prime}} i_{1}\left(w^{\prime}\right) i_{2}\left(w^{\prime \prime}\right) \otimes T^{l\left(w^{\prime}\right)}\left(T_{2}-T\right)^{l\left(w^{\prime \prime}\right)}\right) \\
=\tilde{\Phi}^{(2)}\left(i_{1}(w) i_{2}(1) \otimes T^{n}\right)=\tilde{\Phi}^{(2)}\left(w \otimes 1 \otimes t^{n} \otimes t^{n}\right)=\phi_{1}(w)
\end{gathered}
$$

where we used

$$
\tilde{\Phi}_{2}\left(T^{l\left(w^{\prime}\right)}\left(T_{2}-T\right)^{l\left(w^{\prime \prime}\right)}\right) \neq 0 \text { iff } l\left(w^{\prime \prime}\right)=n \text { and } l\left(w^{\prime \prime}\right)=0,
$$


i.e. the only nonvanishing term is the one for which $w^{\prime}=w, w^{\prime \prime}=1$. The same reasoning can be applied to prove that $\tilde{\Phi}^{(2)} \circ j_{2}^{(2)}=\phi_{2}$.

In the expressions for $j_{k}^{(2)}(w), k=1,2$, there is always exactly one term in which $T$ does not appear. It will play a special role in the sequel, therefore a new notation is desirable. Thus, for words $w, v$ in $\mathcal{A}_{1}, \mathcal{A}_{2}$, respectively, let

$$
\begin{gathered}
d_{1}^{(2)}(w)=i_{2}(w) \otimes T_{2}^{l(w)} \equiv 1 \otimes w \otimes 1 \otimes t^{l(w)}, \\
d_{2}^{(2)}(v)=T_{2}^{l(v)} \otimes i_{2}(v) \equiv 1 \otimes t^{l(v)} \otimes 1 \otimes v .
\end{gathered}
$$

Further, let $\Psi^{(2)}=\mathrm{id} \otimes \tilde{\psi}_{1} \otimes \mathrm{id} \otimes \tilde{\psi}_{2}$. Then it is easy to show that $\left(\Psi^{(2)} \circ d_{1}^{(1)}\right)(w)=\psi_{1}(w) \mathbf{1}$, $\left(\Psi^{(2)} \circ d_{2}^{(1)}\right)(w)=\psi_{2}(v) \mathbf{1}$.

Proposition 4. Let $w, v$ be non-empty words in $\mathcal{A}_{1}, \mathcal{A}_{2}$, respectively. Then

$$
\begin{aligned}
j_{1}^{(2)}(w)-d_{1}^{(2)}(w) & =\sum_{W, R} W \otimes R T, \\
j_{2}^{(2)}(v)-d_{2}^{(2)}(v) & =\sum_{S, T} S T \otimes V
\end{aligned}
$$

where the summations are taken over finite sets of pairs of elementary tensors, $S, R \in$ $\mathbf{C}\left[T, T_{2}\right]$ and $W \in \mathcal{A}_{1} \otimes \mathcal{A}_{1}, V \in \mathcal{A}_{2} \otimes \mathcal{A}_{2}$, respectively.

Proof. Without loss of generality we will prove only the first equation. As we mentioned above, $d_{1}^{(2)}(w)$ appears as one of the elementary tensors in the decomposition of $j_{1}^{(2)}(w)$ given by Proposition 2. Namely, it corresponds to $l\left(w^{\prime}\right)=0$ and $l\left(w^{\prime \prime}\right)=r$. All other terms have a nonzero power of $t$ in the third and fourth tensor sites, thus they assume the given form (the explicit form of the right-hand side can be given, but it is not relevant for our purposes).

We arrive at the crucial factorization property of the 2 -fold tensor product, reminding the one in the conditionally free independence.

LEMMA 5. Let $w_{1}, \ldots, w_{p}$ be non-empty words in $\mathcal{A}_{k_{1}}, \ldots, \mathcal{A}_{k_{n}}$, respectively, where $k_{1}, \ldots, k_{n} \in\{1,2\}$ and $k_{1} \neq k_{2} \neq \ldots \neq k_{n}$. Then

$$
\begin{aligned}
& \tilde{\Phi}^{(2)}\left[\left(j_{k_{1}}^{(2)}\left(w_{1}\right)-d_{k_{1}}^{(2)}\left(w_{1}\right)\right) \ldots\left(j_{k_{n}}^{(2)}\left(w_{n}\right)-d_{k_{n}}^{(2)}\left(w_{n}\right)\right)\right]= \\
& =\left(\phi_{k_{1}}\left(w_{1}\right)-\psi_{k_{1}}\left(w_{1}\right)\right) \ldots\left(\phi_{k_{n}}\left(w_{n}\right)-\psi_{k_{n}}\left(w_{n}\right)\right) .
\end{aligned}
$$

Proof. By linearity it is enough to show the factorization property for elementary tensors appearing in the right-hand sides of the formulas given by Proposition 4. Further, without loss of generality, assume that $k_{1}=1$. Thus

$$
\begin{aligned}
\tilde{\Phi}^{(2)} & {\left[\left(W_{1} \otimes R_{1} T\right)\left(S_{1} T \otimes V_{1}\right)\left(W_{2} \otimes R_{2} T\right)\left(S_{2} T \otimes V_{2}\right) \ldots\right]=} \\
= & \tilde{\Phi}_{1}^{(2)}\left(W_{1} S_{1} T W_{2} S_{2} T \ldots\right) \tilde{\Phi}_{2}^{(2)}\left(R_{1} T V_{1} R_{2} T V_{2} \ldots\right) \\
= & \tilde{\Phi}_{1}^{(2)}\left(W_{1}\right) \tilde{\Phi}_{1}^{(2)}\left(S_{1} T\right) \tilde{\Phi}_{1}^{(2)}\left(W_{2}\right) \tilde{\Phi}_{1}^{(2)}\left(S_{2} T\right) \ldots \times \\
& \times \tilde{\Phi}_{2}^{(2)}\left(R_{1} T\right) \tilde{\Phi}_{2}^{(2)}\left(V_{1}\right) \tilde{\Phi}_{2}^{(2)}\left(R_{2} T\right) \tilde{\Phi}_{2}^{(2)}\left(V_{2}\right) \ldots \\
= & \tilde{\Phi}^{(2)}\left(W_{1} \otimes R_{1} T\right) \tilde{\Phi}^{(2)}\left(S_{1} T \otimes V_{1}\right) \tilde{\Phi}^{(2)}\left(W_{2} \otimes R_{2} T\right) \tilde{\Phi}^{(2)}\left(S_{2} T \otimes V_{2}\right) \ldots
\end{aligned}
$$


Hence, by linearity, we also have

$$
\begin{aligned}
& \tilde{\Phi}^{(2)}\left[\left(j_{k_{1}}^{(2)}\left(w_{1}\right)-d_{k_{1}}^{(2)}\left(w_{1}\right)\right) \ldots\left(j_{k_{n}}^{(2)}\left(w_{n}\right)-d_{k_{n}}^{(2)}\left(w_{n}\right)\right)\right]= \\
& =\tilde{\Phi}^{(2)}\left(j_{k_{1}}^{(2)}\left(w_{1}\right)-d_{k_{1}}^{(2)}\left(w_{1}\right)\right) \ldots \tilde{\Phi}^{(2)}\left(j_{k_{n}}^{(2)}\left(w_{n}\right)-d_{k_{n}}^{(2)}\left(w_{n}\right)\right) .
\end{aligned}
$$

However, by Proposition $3, \tilde{\Phi}^{(2)} \circ j_{k}^{(2)}=\phi_{k}$. Also, $\tilde{\Phi}^{(2)} \circ d_{k}^{(2)}=\psi_{k}, k=1,2$. This finishes the proof.

LEMma 6. Let $\Psi^{(2)}=\mathrm{id} \otimes \tilde{\psi}_{1} \otimes \mathrm{id} \otimes \tilde{\psi}_{2}$ and let $w_{1}, \ldots, w_{n}$ be non-empty words in $\mathcal{A}_{k_{1}}, \ldots, \mathcal{A}_{k_{n}}$, respectively, where $k_{1} \neq k_{2} \neq \ldots \neq k_{n}$. Then $\Psi^{(2)}$ exhibits the following multiplicative property:

$$
\left(\Psi^{(2)} \circ j^{(2)}\right)\left(w_{1} \ldots w_{n}\right)=\left(\Psi^{(2)} \circ j_{k_{1}}^{(2)}\right)\left(w_{1}\right) \ldots\left(\Psi^{(2)} \circ j_{k_{n}}^{(2)}\right)\left(w_{n}\right) .
$$

Proof. Using Proposition 2, it is enough to show the above multiplicative property if $j_{1}^{(2)}\left(w_{k}\right), j_{2}^{(2)}\left(w_{k}\right)$ are replaced by $w_{k}^{\prime} \otimes w_{k}^{\prime \prime} \otimes t_{k}^{\prime} \otimes t_{k}^{\prime \prime}, t_{k}^{\prime} \otimes t_{k}^{\prime \prime} \otimes w_{k}^{\prime} \otimes w_{k}^{\prime \prime}$, respectively, where $t_{k}^{\prime}=t^{l\left(w_{k}\right)-r}, t_{k}^{\prime \prime}=t^{l\left(w_{k}\right)}$ with $0 \leq r \leq l\left(w_{k}^{\prime \prime}\right)$. Since $w_{1}, \ldots, w_{n}$ are nonempty, $l\left(w_{k}\right) \neq 0$, and hence $t_{k}^{\prime \prime} \in t \mathbf{C}[t]$ for $k=1, \ldots, n$, which ensures the separation of words from the same algebra (see the third equality below). Without loss of generality, let $k_{1}=1$. Then

$$
\begin{aligned}
\Psi^{(2)} & {\left[\left(w_{1}^{\prime} \otimes w_{1}^{\prime \prime} \otimes t_{1}^{\prime} \otimes t_{1}^{\prime \prime}\right)\left(t_{2}^{\prime} \otimes t_{2}^{\prime \prime} \otimes w_{2}^{\prime} \otimes w_{2}^{\prime \prime}\right) \times\right.} \\
& \left.\times\left(w_{3}^{\prime} \otimes w_{3}^{\prime \prime} \otimes t_{3}^{\prime} \otimes t_{3}^{\prime \prime}\right)\left(t_{4}^{\prime} \otimes t_{4}^{\prime \prime} \otimes w_{4}^{\prime} \otimes w_{4}^{\prime \prime}\right) \ldots\right] \\
= & \Psi^{(2)}\left[\left(w_{1}^{\prime} t_{2}^{\prime} w_{3}^{\prime} t_{4}^{\prime} \ldots\right) \otimes\left(w_{1}^{\prime \prime} t_{2}^{\prime \prime} w_{3}^{\prime \prime} t_{4}^{\prime \prime} \ldots\right) \otimes\left(t_{1}^{\prime} w_{2}^{\prime} t_{3}^{\prime} w_{4}^{\prime} \ldots\right) \otimes\left(t_{1}^{\prime \prime} w_{2}^{\prime \prime} t_{3}^{\prime \prime} w_{4}^{\prime \prime} \ldots\right)\right] \\
= & \tilde{\psi}_{1}\left(w_{1}^{\prime} t_{2}^{\prime} w_{3}^{\prime} t_{4}^{\prime} \ldots\right) \tilde{\psi}_{2}\left(t_{1}^{\prime} w_{2}^{\prime} t_{3}^{\prime} w_{4}^{\prime} \ldots\right) \times\left(w_{1}^{\prime \prime} t_{2}^{\prime \prime} w_{3}^{\prime \prime} t_{4}^{\prime \prime} \ldots\right) \otimes\left(t_{1}^{\prime \prime} w_{2}^{\prime \prime} t_{3}^{\prime \prime} w_{4}^{\prime \prime} \ldots\right) \\
= & \tilde{\psi}_{1}\left(w_{1}^{\prime}\right) \tilde{\psi}_{1}\left(t_{2}^{\prime}\right) \tilde{\psi}_{1}\left(w_{3}^{\prime}\right) \tilde{\psi}_{1}\left(t_{4}^{\prime}\right) \ldots \tilde{\psi}_{2}\left(t_{1}^{\prime}\right) \tilde{\psi}_{2}\left(w_{2}^{\prime}\right) \tilde{\psi}_{2}\left(t_{3}^{\prime}\right) \tilde{\psi}_{2}\left(w_{4}^{\prime}\right) \ldots \times \\
& \times\left(w_{1}^{\prime \prime} t_{2}^{\prime \prime} w_{3}^{\prime \prime} t_{4}^{\prime \prime} \ldots\right) \otimes\left(t_{1}^{\prime \prime} w_{2}^{\prime \prime} t_{3}^{\prime \prime} w_{4}^{\prime \prime} \ldots\right) \\
= & \Psi^{(2)}\left(w_{1}^{\prime} \otimes w_{1}^{\prime \prime} \otimes t_{1}^{\prime} \otimes t_{1}^{\prime \prime}\right) \Psi^{(2)}\left(t_{2}^{\prime} \otimes t_{2}^{\prime \prime} \otimes w_{2}^{\prime} \otimes w_{2}^{\prime \prime}\right) \times \\
& \times \Psi^{(2)}\left(w_{3}^{\prime} \otimes w_{3}^{\prime \prime} \otimes t_{3}^{\prime} \otimes t_{3}^{\prime \prime}\right) \Psi^{(2)}\left(t_{4}^{\prime} \otimes t_{4}^{\prime \prime} \otimes w_{4}^{\prime} \otimes w_{4}^{\prime \prime}\right) \ldots
\end{aligned}
$$

By linearity, the multiplicative property holds also for $j_{1}^{(2)}\left(w_{k}\right)$ and $j_{2}^{(2)}\left(w_{k}\right)$.

Remark 1. If, in Lemma 6 , we replace any of $j_{k_{i}}^{(2)}\left(w_{i}\right)^{\prime}$ 's by $d_{k_{i}}^{(2)}\left(w_{i}\right)$ 's, then the factorization stated in the theorem still holds. One can go through the proof to see that it is really the case. Actually, in the proof of Theorem 7 we will use this modified version of Lemma 6 , but the multiplicative property of Lemma 6 will be important for future developments, therefore, apart from simplicity arguments, we decided to present the version given above.

TheOrEm 7. Let $\Phi^{(2)}=\Phi_{1}^{(2)} \otimes \Phi_{2}^{(2)}, \Phi_{i}^{(2)}=\tilde{\psi}_{i} \otimes \tilde{\phi}_{i}, i=1,2$, where $\tilde{\psi}_{i}=\psi_{i} *_{B} h$, $\tilde{\phi}_{i}=\phi_{i} *_{B} h$ and $\psi_{i}, \phi_{i}$ are states on the free ${ }^{*}$-algebra $\mathcal{A}_{i}, i=1,2$, and $h$ is $a^{*}$. homomorphism on $\mathbf{C}[t]$ such that $h(t)=1$. Then, $\Phi^{(2)} \circ j^{(2)}$ agrees with $*_{i \in\{1,2\}}\left(\phi_{i}, \psi_{i}\right)$ on words $w_{1} \ldots w_{n}$ for $n \leq 4$

Proof. We have

$$
\begin{aligned}
\tilde{\Phi}^{(2)}\left(j_{k_{1}}^{(2)}\left(w_{1}\right) \ldots j_{k_{n}}^{(2)}\left(w_{n}\right)\right) & =\tilde{\Phi}^{(2)}\left(\left(j_{k_{1}}^{(2)}\left(w_{1}\right)-d_{k_{1}}^{(2)}\left(w_{1}\right)\right) \ldots\left(j_{k_{n}}^{(2)}\left(w_{n}\right)-d_{k_{n}}^{(2)}\left(w_{n}\right)\right)\right) \\
& +\sum_{i} \tilde{\Phi}^{(2)}\left(j_{k_{1}}^{(2)}\left(w_{1}\right) \ldots d_{k_{i}}^{(2)}\left(w_{i}\right) \ldots j_{k_{n}}^{(2)}\left(w_{n}\right)\right)
\end{aligned}
$$




$$
\begin{aligned}
& -\sum_{i<l} \tilde{\Phi}^{(2)}\left(j_{k_{1}}^{(2)}\left(w_{1}\right) \ldots d_{k_{i}}^{(2)}\left(w_{i}\right) \ldots d_{k_{l}}^{(2)}\left(w_{l}\right) \ldots j_{k_{n}}^{(2)}\left(w_{n}\right)\right) \\
& +\ldots-(-1)^{n} \tilde{\Phi}^{(2)}\left(d_{k_{1}}^{(2)}\left(w_{1}\right) \ldots d_{k_{n}}^{(2)}\left(w_{n}\right)\right)
\end{aligned}
$$

for $n \leq 4$. Note that the above relation reminds the one for the conditionally free case, except that instead of numbers we have $d_{k_{i}}^{(2)}(w)$ 's replacing $j_{k_{i}}^{(2)}(w)$ 's at one, two, three or four places. By Lemma 5 it is enough to show that

$$
\begin{aligned}
& \tilde{\Phi}^{(2)}\left(j_{k_{1}}^{(2)}\left(w_{1}\right) \ldots d_{k_{i(1)}}^{(2)}\left(w_{i(1)}\right) \ldots d_{k_{i(l)}}^{(2)}\left(w_{i(l)}\right) \ldots j_{k_{n}}^{(2)}\left(w_{n}\right)\right)= \\
& =*_{i \in\{1,2\}}\left(\phi_{i}, \psi_{i}\right)\left(w_{1} \ldots \breve{w}_{i(1)} \ldots \breve{w}_{i(l)} \ldots w_{n}\right) \psi_{k_{i(1)}}\left(w_{i(1)}\right) \ldots \psi_{k_{i(l)}}\left(w_{i(l)}\right),
\end{aligned}
$$

for $n \leq 4$, where by ${ }^{\smile}$ we understand that the words with indices $i(1), \ldots, i(l)$ are omitted.

We will prove this for the only non-trivial case, i.e. when we have one $d_{k_{i}}^{(2)}\left(w_{i}\right)$ in the middle, i.e. in the second or third place. Without loss of generality we will assume that $i=2$ and $k_{i}=2$. The case $i=2$ and $k_{i}=1$ is similar. In turn, if $i=3$, we obtain the result by mirror reflection. We will use $\tilde{\Phi}^{(2)}=\tilde{\Phi}^{(1)} \circ \Psi_{2}$, although one can also carry out the calculations directly. However, in order to understand better the proofs for $m$-freeness on this model case, we prefer to use the machinery developed in this paper.

In view of Proposition 2, we can write the explicit form of $\left(\Psi^{(2)} \circ j_{k_{i}}^{(2)}\right)\left(w_{i}\right)$ :

$$
\begin{aligned}
\left(\Psi^{(2)} \circ j_{k_{i}}^{(2)}\right)\left(w_{i}\right) & =\sum_{w_{i}^{\prime}, w_{i}^{\prime \prime}} \sum_{r=0}^{l\left(w_{i}^{\prime \prime}\right)}(-1)^{l\left(w_{i}^{\prime \prime}\right)-r}\left(\begin{array}{c}
l\left(w_{i}^{\prime \prime}\right) \\
r
\end{array}\right) \psi_{1}\left(w_{i}^{\prime \prime}\right) w_{i}^{\prime} \otimes t^{l\left(w_{i}^{\prime}\right)+l\left(w_{i}^{\prime \prime}\right)-r} \\
& =\sum_{w_{i}^{\prime}, w_{i}^{\prime \prime}} \psi_{1}\left(w_{i}^{\prime \prime}\right) w_{i}^{\prime} \otimes(1-t)^{l\left(w_{i}^{\prime \prime}\right)} t^{l\left(w_{i}^{\prime}\right)}
\end{aligned}
$$

if $k_{i}=1$ and $w_{i} \in \mathcal{A}_{1}$, and

$$
\left(\Psi^{(2)} \circ j_{k_{i}}^{(2)}\right)\left(w_{i}\right)=\sum_{w_{i}^{\prime}, w_{i}^{\prime \prime}} \psi_{2}\left(w_{i}^{\prime \prime}\right)(1-t)^{l\left(w_{i}^{\prime \prime}\right)} t^{l\left(w_{i}^{\prime}\right)} \otimes w_{i}^{\prime}
$$

if $k_{i}=2$ and $w_{i} \in \mathcal{A}_{2}$ for some $i$, The terms which correspond to $l\left(w_{i}^{\prime \prime}\right)=0$ are equal to $w_{i} \otimes t^{l\left(w_{i}\right)}, t^{l\left(w_{i}\right)} \otimes w_{i}$, respectively. In turn, all terms, for which $0<l\left(w_{i}^{\prime \prime}\right)<l\left(w_{i}\right)$, belong to one of the two two-sided ideals in $\tilde{\mathcal{A}}_{1} \otimes \tilde{\mathcal{A}}_{2}, L_{1}$ or $L_{2}$, generated by $1 \otimes(1-t) t$ and $(1-t) t \otimes 1$, respectively. Note that $L_{1}, L_{2} \subset \operatorname{ker} \tilde{\phi}_{1} \otimes \tilde{\phi}_{2}$. Thus, if $k_{i}=1$ and $w_{i} \in \mathcal{A}_{1}$, we can write

$$
\left(\Psi^{(2)} \circ j_{1}^{(2)}\right)\left(w_{i}\right)=\tilde{w}_{i} \quad\left(\bmod L_{1}\right)
$$

where

$$
\tilde{w}_{i}=w_{i} \otimes t^{l\left(w_{i}\right)}+\psi_{1}\left(w_{i}\right) 1 \otimes(1-t)^{l\left(w_{i}\right)} .
$$

Similarly, if $k_{i}=2$ and $w_{i} \in \mathcal{A}_{2}$, we obtain

$$
\left(\Psi^{(2)} \circ j_{2}^{(2)}\right)\left(w_{i}\right)=\tilde{w}_{i}\left(\bmod L_{2}\right),
$$

where

$$
\tilde{w}_{i}=t^{l\left(w_{i}\right)} \otimes w_{i}+\psi_{2}\left(w_{i}\right)(1-t)^{l\left(w_{i}\right)} \otimes 1 .
$$

We can now use the above relations as well as Remark 1 to see that 


$$
\begin{aligned}
& \Phi^{(2)}\left(j_{1}^{(2)}\left(w_{1}\right) d_{2}^{(2)}\left(w_{2}\right) j_{1}^{(2)}\left(w_{3}\right) j_{2}^{(1)}\left(w_{4}\right)\right)= \\
& =\psi_{2}\left(w_{2}\right)\left(\tilde{\phi}_{1} \otimes \tilde{\phi}_{2}\right)\left(\tilde{w}_{1} \tilde{w}_{3} \tilde{w}_{4}\right) \\
& =\psi_{2}\left(w_{2}\right)\left(\tilde{\phi}_{1} \otimes \tilde{\phi}_{2}\right)\left(\left(w_{1} \otimes t^{l\left(w_{1}\right)}\right)\left(w_{3} \otimes t^{l\left(w_{3}\right)}+\psi_{1}\left(w_{3}\right) 1 \otimes(1-t)^{l\left(w_{3}\right)}\right)\left(t^{l\left(w_{4}\right)} \otimes w_{4}\right)\right) \\
& =\psi_{2}\left(w_{2}\right) \phi_{1}\left(w_{1} w_{3}\right) \phi_{2}\left(w_{4}\right)=\psi_{2}\left(w_{2}\right) \times *_{i \in\{1,2\}}\left(\phi_{i}, \psi_{i}\right)\left(w_{1} w_{3} w_{4}\right) .
\end{aligned}
$$

The other cases can be verified immediately (actually, the one that we have verified is the only one that requires some calculations). This finishes the proof.

Remark 2. Of course, one would like to give an explicit formula for all correlations of 2 -freeness. This can be done in terms of diagrams, but the combinatorial techniques that need to be developed are quite extensive and go beyond the scope of this article, thus will be presented elsewhere.

Remark 3. It can be shown that if we take $n=5$, then $\tilde{\Phi}^{(2)} \circ j^{(2)}$ does not agree with the conditionally free product. Then one needs to take the third order approximation of freeness in the framework discussed in Section 6.

4. An extension to infinitely many free *-algebras. An extension of the construction presented in Section 3 to the case of infinitely many free ${ }^{*}$-algebras is very natural. Let $\mathcal{A}_{i}, i \in \mathbf{N}$, be a family of free ${ }^{*}$-algebras generated by $\mathcal{G}_{i}^{+}$. As before, denote by $\tilde{\mathcal{A}}_{i}=\mathcal{A}_{i} * \mathbf{C}[t]$ the free product of $\mathcal{A}_{i}$ and the algebra of polynomials in one variable $t$. For each $i \in \mathbf{N}$ we identify the units of $\mathcal{A}_{i}$ and $\mathbf{C}[t]$ and, by abuse of notation, we denote the unit of each product by 1 . As before, take Boolean extensions $\tilde{\phi}_{i}, \tilde{\phi}_{i}$ on $\tilde{\mathcal{A}}_{i}$, respectively, where $\tilde{\phi}_{i}=\phi_{i} * h, \tilde{\psi}_{i}=\psi_{i} * h, h$ is a ${ }^{*}$-homomorphism on $\mathbf{C}[t]$ and $h(t)=1, i \in \mathbf{N}$. In the free product $*_{i \in \mathbf{N}} \mathcal{A}_{i}$ we identify the units of $\mathcal{A}_{i}, i \in \mathbf{N}$. By abuse of notation, we denote $\mathbf{1}=1^{\otimes \infty}$.

Definition 3. For given $a \in \mathcal{G}_{i}$, let

$$
j_{i}^{(2)}(a)=T^{\otimes(i-1)} \otimes i_{1}(a) \otimes T^{\otimes \infty}+T_{2}^{\otimes(i-1)} \otimes i_{2}(a) \otimes T_{2}^{\otimes \infty}-T^{\otimes(n-1)} \otimes i_{2}(a) \otimes T^{\otimes \infty},
$$

and define the ${ }^{*}$-homomorphism

$$
j^{(2)}: \quad *_{i \in \mathbf{N}} \mathcal{A}_{i} \rightarrow \bigotimes_{i \in \mathbf{N}} \tilde{\mathcal{A}}_{i}^{\otimes 2}
$$

as the linear extension of $j^{(2)}(1)=1$ and

$$
j^{(2)}\left(w_{1} \ldots w_{n}\right)=j_{k_{1}}^{(2)}\left(w_{1}\right) \ldots j_{k_{n}}^{(2)}\left(w_{n}\right)
$$

where $w_{1}, \ldots, w_{n}$ are non-empty words in $\mathcal{A}_{k_{1}}, \ldots, \mathcal{A}_{k_{n}}$ with $k_{1}, \ldots k_{n} \in \mathbf{N}$.

There is no simple analogue of Proposition 1, therefore we start with the analogue of Proposition 2. A direct proof will be presented.

Proposition 8. Let $w$ be a non-empty word in $\mathcal{A}_{i}$. Then

$$
\begin{aligned}
j_{i}^{(2)}(w) & =\sum_{w^{\prime}, w^{\prime \prime}} \sum_{r=0}^{l\left(w^{\prime \prime}\right)}\left(\begin{array}{c}
l\left(w^{\prime \prime}\right) \\
r
\end{array}\right)(-1)^{l\left(w^{\prime \prime}\right)-r}\left(t^{l(w)-r} \otimes t^{l(w)}\right)^{\otimes(i-1)} \otimes \\
& \otimes w^{\prime} \otimes w^{\prime \prime} \otimes\left(t^{l(w)-r} \otimes t^{l(w)}\right)^{\otimes \infty} .
\end{aligned}
$$


Proof. Let $w=a_{1} \ldots a_{n}$. Then

where

$$
j_{i}^{(2)}(w)=\prod_{k=1}^{n}\left(j_{i, 1}^{(2)}\left(a_{k}\right)+j_{i, 2}^{(2)}\left(a_{k}\right)-j_{i, 3}^{(2)}\left(a_{k}\right)\right)
$$

$$
\begin{gathered}
j_{i, 1}^{(2)}\left(a_{k}\right)=T^{\otimes(i-1)} \otimes i_{1}\left(a_{k}\right) \otimes T^{\otimes \infty}, \quad j_{i, 2}^{(2)}\left(a_{k}\right)=T_{2}^{\otimes(i-1)} \otimes i_{2}\left(a_{k}\right) \otimes T_{2}^{\otimes \infty} \\
j_{i, 3}^{(2)}\left(a_{k}\right)=T^{\otimes(i-1)} \otimes i_{2}\left(a_{k}\right) \otimes T^{\otimes \infty} .
\end{gathered}
$$

We obtain

$$
j_{i}^{(2)}(w)=\sum_{p_{1}, \ldots, p_{n} \in\{1,2,3\}}(-1)^{\left|J_{3}\right|} j_{i, p_{1}}^{(2)}\left(a_{1}\right) \ldots j_{i, p_{n}}^{(2)}\left(a_{n}\right)
$$

where $J_{k}=\left\{m \mid p_{m}=k\right\}, k=1,2,3$. We arrive at the formula

$$
\begin{gathered}
j_{i}^{(2)}(w)=\sum_{p_{1}, \ldots, p_{n} \in\{1,2,3\}}(-1)^{\left|J_{3}\right|}\left(T^{\left|J_{1} \cup J_{3}\right|} T_{2}^{\left|J_{2}\right|}\right)^{\otimes(i-1)} \otimes \\
\otimes i_{1}\left(w_{J_{1}}\right) i_{2}\left(w_{J_{2} \cup J_{3}}\right) \otimes\left(T^{\left|J_{1} \cup J_{3}\right|} T_{2}^{\left|J_{2}\right|}\right)^{\otimes \infty} .
\end{gathered}
$$

We will now translate the sum into our notation from Section 3. Denote $w^{\prime}=w_{J_{1}}$, $w^{\prime \prime}=w_{J_{2} \cup J_{3}}$. Then $l(w)=\left|J_{1}\right|+\left|J_{2}\right|+\left|J_{3}\right|, l\left(w^{\prime}\right)=\left|J_{1}\right|, l\left(w^{\prime \prime}\right)=\left|J_{2}\right|+\left|J_{3}\right|$. Note that $w^{\prime}$ is in one-to-one correspondence with $J_{1}$. However, to each $w^{\prime}, w^{\prime \prime}$, and $0 \leq r \leq l\left(w^{\prime \prime}\right)$ there are $\left(\begin{array}{c}l\left(w^{\prime \prime}\right) \\ r\end{array}\right)$ different tuples $\left(k_{1}, \ldots, k_{n}\right)$ which contribute the term

$$
(-1)^{l\left(w^{\prime \prime}\right)-r}\left(t^{l(w)-r} \otimes t^{l(w)}\right)^{\otimes(i-1)} \otimes w^{\prime} \otimes w^{\prime \prime} \otimes\left(t^{l(w)-r} \otimes t^{l(w)}\right)^{\otimes \infty},
$$

which finishes the proof.

Before we go on, let us define

$$
d_{i}^{(2)}(w)=\left(T_{2}^{l(w)}\right)^{\otimes(i-1)} \otimes i_{2}(w) \otimes\left(T_{2}^{l(w)}\right)^{\otimes \infty}
$$

where $w$ is a non-empty word in $\mathcal{A}_{i}$.

Proposition 9. Let $w$ be a non-empty word in $\mathcal{A}_{n}$. Then

$$
j_{i}^{(2)}(w)-d_{i}^{(2)}(w)=\sum_{R, W}(R T)^{\otimes(i-1)} \otimes W \otimes(R T)^{\otimes \infty}
$$

where $R, W$ are elementary tensors in $\mathbf{C}\left[T, T_{2}\right], \mathcal{A}_{n} \otimes \mathcal{A}_{n}$, respectively.

Proof. In the expression for $j_{i}^{(2)}(w)$ given by Proposition 8 there is only one elementary tensor which does not have $t$ in all tensor sites lying to the right and to the left of the $2 i-1$-th and $2 i$-th sites. This term is exactly equal to $d_{i}^{(2)}(w)$. Therefore, if we subtract it, the remaining terms are of the desired form.

We introduce the infinite tensor product versions of $\tilde{\Phi}^{(2)}$ and $\Psi^{(2)}$ of Section 3 , by abuse of notation denoting them

$$
\tilde{\Phi}^{(2)} \equiv \bigotimes_{i \in \mathbf{N}} \tilde{\Phi}_{i}^{(2)}=\bigotimes_{i \in \mathbf{N}}\left(\tilde{\phi}_{i} \otimes \tilde{\psi}_{i}\right), \quad \Psi^{(2)}=\bigotimes_{i \in \mathbf{N}}\left(\mathrm{id} \otimes \tilde{\psi}_{i}\right)
$$

where $\tilde{\psi}_{i}(t)=\tilde{\phi}_{i}(t)=h(t)=1$. Then it is easy to see that $\tilde{\Phi}^{(2)} \circ j_{i}^{(2)}=\phi_{i}$ and $\Psi^{(2)} \circ d_{i}^{(2)}=$ $\psi_{i} \mathbf{1}, i \in \mathbf{N}$. 
LEMma 10. Let $w_{1}, \ldots, w_{n}$ be non-empty words in $\mathcal{A}_{k_{1}}, \ldots, \mathcal{A}_{k_{n}}$, respectively, where $k_{1} \neq k_{2} \neq \ldots \neq k_{n}$. Then

$$
\begin{aligned}
& \tilde{\Phi}^{(2)}\left[\left(j_{k_{1}}^{(2)}\left(w_{1}\right)-d_{k_{1}}^{(2)}\left(w_{1}\right)\right) \ldots\left(j_{k_{n}}^{(2)}\left(w_{n}\right)-d_{k_{n}}^{(2)}\left(w_{n}\right)\right)\right] \\
& =\left(\phi_{k_{1}}\left(w_{1}\right)-\psi_{k_{1}}\left(w_{1}\right)\right) \ldots\left(\phi_{k_{n}}\left(w_{n}\right)-\psi_{k_{n}}\left(w_{n}\right)\right) .
\end{aligned}
$$

Pro of. It is enough to show the factorization for the elementary tensors of Proposition 9. We order $k_{1}, \ldots, k_{n}$ so that $\left\{k_{1}, \ldots, k_{n}\right\}=\left\{r_{1}, \ldots, r_{l}\right\}$ and $r_{1}<\ldots<r_{l}, l \leq n$. Then

$$
\begin{aligned}
& \tilde{\Phi}^{(2)}\left[\prod_{i=1}^{n}\left(\left(R_{i} T\right)^{\otimes\left(k_{i}-1\right)} \otimes W_{i} \otimes\left(R_{i} T\right)^{\otimes \infty}\right)\right]= \\
& =\prod_{m=1}^{l}\left(\prod_{j \in P_{m}} \tilde{\Phi}_{r_{m}}^{(2)}\left(W_{j}\right) \prod_{j \notin P_{m}} \tilde{\Phi}_{r_{m}}^{(2)}\left(R_{j} T\right)\right) \prod_{j \notin\left\{r_{1}, \ldots, r_{l}\right\}} \prod_{i=1, \ldots, n} \tilde{\Phi}_{j}^{(2)}\left(R_{i} T\right) \\
& =\prod_{i=1}^{n} \tilde{\Phi}^{(2)}\left[\left(R_{i} T\right)^{\otimes\left(k_{i}-1\right)} \otimes W_{i} \otimes\left(R_{i} T\right)^{\otimes \infty}\right],
\end{aligned}
$$

where $P=\left\{P_{1}, \ldots, P_{l}\right\}$ is the partition of $\{1, \ldots, n\}$ associated with $\left\{k_{1}, \ldots, k_{n}\right\}$, given by $P_{m}=\left\{s \mid k_{s}=r_{m}\right\}, m=1, \ldots, l$. Use Proposition 9 to get the final result.

Lemma 11. Let $\Psi^{(2)}=\bigotimes_{i \in \mathbf{N}}\left(\tilde{\psi}_{i} \otimes\right.$ id $)$ and let $w_{1}, \ldots, w_{n}$ be non-empty words in $\mathcal{A}_{k_{1}}, \ldots, \mathcal{A}_{k_{n}}$, respectively, where $k_{1} \neq k_{2} \neq \ldots, \neq k_{n}$. Then $\Psi^{(2)}$ has the following multiplicative property:

$$
\left(\Psi^{(2)} \circ j^{(2)}\right)\left(w_{1} \ldots w_{n}\right)=\left(\Psi^{(2)} \circ j_{k_{1}}^{(2)}\right)\left(w_{1}\right) \ldots\left(\Psi^{(2)} \circ j_{k_{n}}^{(2)}\right)\left(w_{n}\right)
$$

Proof. By Proposition 8 it is enough to show the factorization for products of elementary tensors of the form

$$
\left(t_{m}^{\prime} \otimes t_{m}^{\prime \prime}\right)^{k_{m}-1} \otimes w_{m}^{\prime} \otimes w_{m}^{\prime \prime} \otimes\left(t_{m}^{\prime} \otimes t_{m}^{\prime \prime}\right)^{\otimes \infty},
$$

$m=1, \ldots, n$. As in the proof of Lemma 3.7, this boils down to showing the appropriate factorization for $\psi_{i}, i \in \mathbf{N}$. Namely, if in the free product $w_{1} \ldots w_{n}$, words $w_{j}, w_{k}$, where $j<k$, belong to the same algebra, say $\mathcal{A}_{p}$, and are separated by words from other algebras, then at the $2 p$-th site they are separated by $t^{l\left(w_{j+1}\right)+\ldots+l\left(w_{k-1}\right)}$. Applying $\Psi^{(2)}$ to this site produces

$$
\begin{aligned}
& \psi_{p}\left(\ldots t^{l\left(w_{j-1}\right)} w_{j}^{\prime \prime} t^{l\left(w_{j+1}\right)+\ldots+l\left(w_{k-1}\right)} w_{k}^{\prime \prime} t^{l\left(w_{k+1}\right)} \ldots\right) \\
& =\psi_{p}\left(\ldots t^{l\left(w_{j-1}\right)}\right) \psi_{p}\left(w_{j}^{\prime \prime}\right) \psi_{p}\left(t^{l\left(w_{j+1}\right)}\right) \ldots \psi_{p}\left(t^{l\left(w_{k-1}\right)}\right) \psi_{p}\left(w_{k}^{\prime \prime}\right) \psi_{p}\left(t^{l\left(w_{k+1}\right)} \ldots\right),
\end{aligned}
$$

which implies the desired factorization for $\Psi^{(2)}$

THEOREM 12. Let $\tilde{\Phi}^{(2)}=\bigotimes_{i \in \mathbf{N}} \tilde{\Phi}_{i}^{(2)}, \tilde{\Phi}_{i}^{(2)}=\tilde{\psi}_{i} \otimes \tilde{\phi}_{i}$, where $\tilde{\psi}_{i}=\psi_{i} *_{B} h, \tilde{\phi}_{i}=\phi_{i} *_{B} h$ and $\psi_{i}, \phi_{i}$ are states on the free ${ }^{*}$-algebra $\mathcal{A}_{i}, i \in \mathbf{N}$, and $h$ is a ${ }^{*}$-homomorphism from $\mathbf{C}[t]$ into $\mathbf{C}$ such that $h(t)=1$. Then, $\tilde{\Phi}^{(2)} \circ j^{(2)}$ agrees with $*_{i \in \mathbf{N}} \mathcal{A}_{i}$ on words $w_{1} \ldots w_{n}$ for $n \leq 4$.

Pr o of. The proof is similar to that of Theorem 7 (Lemmas 10-11 are used). 
5. Construction of the associated *-bialgebra. We would like to associate a *bialgebra with 2 -freeness. It seems that one should be able to do that using a ${ }^{*}$-subalgebra of the double tensor product $\tilde{\mathcal{A}} \otimes \tilde{\mathcal{A}}$. Nevertheless, it turns out that one needs to take the free product ${ }^{*}$-bialgebra $\tilde{\mathcal{A}}^{*(2)} \equiv \tilde{\mathcal{A}} * \tilde{\mathcal{A}}$ instead. The construction of this ${ }^{*}$-bialgebra is presented below. First, we must introduce a free version of the ${ }^{*}$-homomorphism $j^{(2)}$.

Definition 4. For given $a \in \mathcal{G}_{1}, b \in \mathcal{G}_{2}$, let $a_{(1)}, a_{(2)}$ denote different copies of $a$ in $\tilde{\mathcal{A}}_{1} * \tilde{\mathcal{A}}_{1}, b_{(1)}, b_{(2)}$ denote different copies of $b$ in $\tilde{\mathcal{A}}_{2} * \tilde{\mathcal{A}}_{2}$, and $t_{(1)}, t_{(2)}$ stand for different copies of $t$ in both products. Let

$$
\begin{aligned}
& \hat{j}_{1}^{(2)}(a)=a_{(1)} \otimes t_{(1)} t_{(2)}+a_{(2)} \otimes\left(t_{(1)}-t_{(1)} t_{(2)}\right), \\
& \hat{j}_{2}^{(2)}(b)=t_{(1)} t_{(2)} \otimes b_{(1)}+\left(t_{(1)}-t_{(1)} t_{(2)}\right) \otimes b_{(2)}
\end{aligned}
$$

and define the ${ }^{*}$-homomorphism

$$
\hat{j}^{(2)}: \mathcal{A}_{1} * \mathcal{A}_{2} \rightarrow\left(\tilde{\mathcal{A}}_{1} * \tilde{\mathcal{A}}_{1}\right) \otimes\left(\tilde{\mathcal{A}}_{2} * \tilde{\mathcal{A}}_{2}\right)
$$

as the linear extension of $\hat{j}^{(2)}(1)=1 \otimes 1$ and

$$
\hat{j}^{(2)}\left(w_{1} \ldots w_{n}\right)=\hat{j}_{k_{1}}^{(2)}\left(w_{1}\right) \ldots \hat{j}_{k_{n}}^{(2)}\left(w_{n}\right),
$$

where $w_{1}, \ldots, w_{n}$ are non-empty words in $\mathcal{A}_{k_{1}}, \ldots, \mathcal{A}_{k_{n}}$, where $k_{1}, \ldots, k_{n} \in\{1,2\}$.

Note that if we take $a_{(1)}=a \otimes 1, a_{(2)}=1 \otimes a, b_{(1)}=b \otimes 1, b_{(2)}=1 \otimes b, t_{(1)}=t \otimes 1$, $t_{(2)}=1 \otimes t$, we obtain Definition 2 .

Let $\mathcal{A}_{1}=\mathcal{A}_{2}=\mathcal{A}$ in the above definition. We can associate a cocommutative *bialgebra with the pair $(\mathcal{A}, \hat{j})$. This is done in the theorem below which also shows how close $\hat{j}^{(2)} \circ \delta$ is to a coproduct.

TheOrem 13. The ${ }^{*}$-algebra $\tilde{\mathcal{A}}^{*(2)}$ can be equipped with the coproduct

$$
\begin{gathered}
\Delta^{(2)}(1)=1 \otimes 1, \quad \Delta^{(2)}\left(t_{(1)}\right)=t_{(1)} \otimes t_{(1)}, \quad \Delta^{(2)}\left(t_{(2)}\right)=t_{(2)} \otimes t_{(2)}, \\
\Delta^{(2)}\left(a_{(2)}\right)=a_{(2)} \otimes t_{(2)}+t_{(2)} \otimes a_{(2)}, \quad \Delta^{(2)}\left(a_{(0)}\right)=a_{(0)} \otimes t_{(1)} t_{(2)}+t_{(1)} t_{(2)} \otimes a_{(0)},
\end{gathered}
$$

and the counit

$$
\epsilon^{(2)}\left(t_{(1)}\right)=\epsilon^{(2)}\left(t_{(2)}\right)=\epsilon^{(2)}(1)=1, \quad \epsilon^{(2)}\left(a_{(1)}\right)=\epsilon^{(2)}\left(a_{(0)}\right)=0
$$

where $a_{(0)}=a_{(1)}-a_{(2)}$ and $a_{(1)}, a_{(2)}$ are different copies of $a \in \mathcal{G}$ in $\tilde{\mathcal{A}}^{*(2)}$. Moreover, if $\delta: \mathcal{A} \rightarrow \mathcal{A} * \mathcal{A}$ is the ${ }^{*}$-homomorphism defined by $\delta(1)=1, \delta(a)=a^{\prime}+a^{\prime \prime}$, where $a^{\prime}, a^{\prime \prime}$ are different copies of a in $\mathcal{A} * \mathcal{A}$, then

$$
\hat{j}^{(2)} \circ \delta=\Delta^{(2)} \circ \hat{i}_{1}
$$

where $\hat{i}_{1}: \mathcal{A} \rightarrow \tilde{\mathcal{A}}^{*(2)}$ is the canonical ${ }^{*}$-homomorphic embedding given by $\hat{i}_{1}(a)=a_{(1)}$.

Proof. It is easy to check that $\Delta^{(2)}$ is coassociative and that $\epsilon^{(2)}$ is the counit. Therefore $\left(\mathcal{A}^{*(2)}, \Delta^{(2)}, \epsilon^{(2)}\right)$ becomes a *-bialgebra.

Now, $\left(\hat{j}^{(2)} \circ \delta\right)(1)=1 \otimes 1=\left(\Delta^{(2)} \circ \hat{i}_{1}(1)\right.$. If $a \in \mathcal{G}$, then

$$
\begin{aligned}
\left(\hat{j}^{(2)} \circ \delta\right)(a)= & \hat{j}_{1}^{(2)}(a)+\hat{j}_{2}^{(2)}(a) \\
= & a_{(1)} \otimes t_{(1)} t_{(2)}+t_{(1)} t_{(2)} \otimes a_{(1)}+a_{(2)} \otimes t_{(2)}+ \\
& +t_{(2)} \otimes a_{(2)}-a_{(2)} \otimes t_{(1)} t_{(2)}-t_{(1)} t_{(2)} \otimes a_{(2)} \\
= & \Delta\left(a_{(1)}\right)=\left(\Delta \circ \hat{i}_{1}\right)(a) .
\end{aligned}
$$


This implies that this identity holds also for arbitrary words in $\mathcal{A}$ since $\hat{j}^{(2)}, \delta, \Delta^{(2)}$ and $\hat{i}_{1}$ are ${ }^{*}$-homomorphisms.

One can say that $\hat{j}^{(2)} \circ \delta$ agrees with the coproduct $\Delta^{(2)}$ restricted to the ${ }^{*}$-subalgebra $\hat{i}_{1}(\mathcal{A})$. Note that $\Delta^{(2)}$ takes $\hat{i}_{1}(\mathcal{A})$ out of $\hat{i}_{1}(\mathcal{A}) \otimes \hat{i}_{1}(\mathcal{A})$ and that is the reason why we have to take $\tilde{\mathcal{A}}^{*(2)}$ and one copy of $\mathcal{A}$ is not enough. Moreover, note that the coproduct defined above preserves the relation $t_{(1)} t_{(2)}=t_{(2)} t_{(1)}$, but it does not preserve $a_{(1)} \tilde{a}_{(2)}=\tilde{a}_{(2)} a_{(1)}$. That is why we have to take the free product $\tilde{\mathcal{A}}^{*(2)}$ instead of the tensor product $\tilde{\mathcal{A}}^{\otimes 2}$ which, at least at the first sight, seemed natural. In other words, in order to be able to associate a *-bialgebra with 2-freeness, we have to resign from the tensor product of different copies of $\tilde{\mathcal{A}}$ in favor of the free product. Thus, we can lift $\tilde{\Phi}^{(2)}$ to $\tilde{\mathcal{A}}^{*(2)} \otimes \tilde{\mathcal{A}}^{*(2)}$ or to $\tilde{\mathcal{A}}^{*(2)} / \mathcal{T}_{0} \otimes \tilde{\mathcal{A}}^{*(2)} / \mathcal{T}_{0}$, where $\mathcal{T}_{0}$ is the two-sided ideal generated by

$$
t_{(1)} t_{(2)}-t_{(2)} t_{(1)}, \quad t_{(1)} a_{(2)}-a_{(2)} t_{(1)}, \quad t_{(2)} a_{(1)}-a_{(1)} t_{(2)},
$$

where $a \in \mathcal{G}$, since $\mathcal{T}_{0}$ is a coideal $\left(\Delta^{(2)}\right.$ preserves the above relations).

Let us look now at the convolutions of states. It is known how to define convolutions of states for *-bialgebras. Namely, if $\Phi_{1}, \Phi_{2}$ are two states on a *-bialgebra $(\mathcal{B}, \Delta, \epsilon)$, then the convolution of $\Phi_{1}$ and $\Phi_{2}$ is given by

$$
\Phi_{1} \star \Phi_{2} \equiv\left(\Phi_{1} \otimes \Phi_{2}\right) \circ \Delta .
$$

In turn, the conditionally free convolution of states on $\mathcal{A}$ is a state on $\mathcal{A}$ given by

$$
\left(\phi_{1}, \psi_{1}\right) \star\left(\phi_{2}, \psi_{2}\right)=*_{i \in\{1,2\}}\left(\phi_{i}, \psi_{i}\right) \circ \delta .
$$

Thus, Theorem 7 enables us to give the second order approximation of the conditionally free convolution of states on a given free ${ }^{*}$-algebra $\mathcal{A}$ in terms of the convolution of states on the ${ }^{*}$-bialgebra $\left(\tilde{\mathcal{A}}^{*(2)}, \Delta^{(2)}, \epsilon^{(2)}\right)$.

For that purpose, we lift the states $\tilde{\Phi}_{k}^{(2)}, k=1,2$, defined on $\tilde{\mathcal{A}}^{\otimes 2}$ to states on $\tilde{\mathcal{A}}^{*(2)}$. By abuse of notation we also denote them by $\tilde{\Phi}_{k}^{(2)}, k=1,2$. Then we can conclude that $\left(\tilde{\Phi}_{1} \star \tilde{\Phi}_{2}\right) \circ \hat{i}_{1}$ agrees with $\left(\phi_{1}, \psi_{1}\right) \star\left(\phi_{2}, \psi_{2}\right)$ on products $w_{1} \ldots w_{n}$ for $n \leq 4$.

Successive iterations of $\delta$ and $\Delta$ can be defined in order to study convolutions of higher order in a manner analogous to the one presented above.

6. Hierarchy of freeness. The approach to freeness via tensor product constructions can be generalized and successive approximations of freeness can be defined. We discuss it in this section for two free ${ }^{*}$-algebras, but an extension to infinitely many or even uncountably many free ${ }^{*}$-algebras presents no difficulty.

Thus, for each $m \in \mathbf{N}$ take as the quantum probability space the pair $\left(\tilde{\mathcal{A}}^{(m)}, \tilde{\Phi}^{(m)}\right)$, where

and

$$
\tilde{\mathcal{A}}^{(m)} \equiv \tilde{\mathcal{A}}_{1}^{\otimes m} \otimes \tilde{\mathcal{A}}_{2}^{\otimes m}
$$

$\tilde{\Phi}^{(m)} \equiv \tilde{\Phi}_{1}^{(m)} \otimes \tilde{\Phi}_{2}^{(m)}$,

where

$$
\tilde{\Phi}_{k}^{(m)}=\tilde{\phi}_{k} \otimes \tilde{\psi}_{k}^{\otimes(m-1)}
$$


and $k=1,2$. Then, one can define the corresponding sequence $j^{(m)}$ of *-homomorphisms as follows.

For given $a \in \mathcal{G}_{1}, b \in \mathcal{G}_{2}$ and $m \in \mathbf{N}$ let

$$
\begin{aligned}
& j_{1}^{(m)}(a)=\sum_{k=1}^{m}\left(i_{k, m}(a)-i_{k+1, m}(a)\right) \otimes t_{[k, m]}, \\
& j_{2}^{(m)}(b)=\sum_{k=1}^{m} t_{[k, m]} \otimes\left(i_{k, m}(b)-i_{k+1, m}(b)\right)
\end{aligned}
$$

where $t_{[k, m]}=I_{k-1} \otimes t^{\otimes(m-k+1)}, i_{k, m}(a)=I_{k-1} \otimes a \otimes I_{m-k}$, where $I_{k}=1^{\otimes k}$. Define the ${ }^{*}$-homomorphism

$$
j^{(m)}: \mathcal{A}_{1} * \mathcal{A}_{2} \rightarrow \tilde{\mathcal{A}}^{(m)}
$$

as the linear extension of $j^{(m)}(1)=I_{m} \otimes I_{m}$ and

$$
j^{(m)}\left(w_{1} \ldots w_{n}\right)=j_{k_{1}}^{(m)}\left(w_{1}\right) \ldots j_{k_{n}}^{(m)}\left(w_{n}\right),
$$

where $w_{1}, \ldots, w_{n}$ are non-empty words in $\mathcal{A}_{k_{1}}, \ldots, \mathcal{A}_{k_{n}}$, where $k_{1}, \ldots, k_{n} \in\{1,2\}$.

Then generalizations of Theorems 7 and 12 can be proven to hold. Namely, one can show that $\tilde{\Phi} \circ j^{(m)}$ agrees with the conditionally free product on words $w_{1} \ldots w_{n}$, where $n \leq 2 m$. Thus we obtain a hierarchy of freeness in the sense that we have a sequence of quantum probability spaces that are closer and closer to the conditionally free product of ${ }^{*}$-algebras. Moreover, we can embed all those spaces in the infinite tensor product $\tilde{\mathcal{A}}_{1}^{\otimes \infty} \otimes \tilde{\mathcal{A}}_{2}^{\otimes \infty}$ with the infinite tensor product of states $\tilde{\Phi} \equiv \tilde{\Phi}_{1} \otimes \tilde{\Phi}_{2}$, where

$$
\tilde{\Phi}_{k}=\tilde{\phi}_{k} \otimes \tilde{\psi}_{k}^{\otimes \infty}
$$

and thus obtain one quantum probability space in which each $m$-freeness lives. In this framework, freeness will correspond to $m=\infty$. Details of this approach will be presented elsewhere.

\section{References}

[B-L-S] M. Bożejko, M. Leinert and R. Speicher, Convolution and limit theorems for conditionally free random variables, Pac. J. Math. 175, No.2 (1996), 357-388.

[Len1] R. Lenczewski, On sums of q-independent $S U_{q}(2)$ quantum variables, Comm. Math. Phys. 154 (1993), 127-134.

[Len2] R. Lenczewski, Addition of independent variables in quantum groups, Rev. Math. Phys. 6 (1994), 135-147.

[Sch] M. Schürmann, White Noise on Bialgebras, Springer-Verlag, Berlin, 1993.

[V-D-N] D. V. Voiculescu, K. J. Dykema and A. Nica, Free Random Variables, CRM Monograph Series, AMS, Providence, 1992. 